\title{
Expansion of Internal Hyphal Growth in Fusarium Head Blight-Infected Grains Contributes to the Elevated Mycotoxin Production During the Malting Process
}

\author{
Zhao Jin, ${ }^{1}$ Shyam Solanki, ${ }^{2,3, \dagger}$ Gazala Ameen, ${ }^{2,3}$ Thomas Gross, ${ }^{3}$ Roshan Sharma Poudel, ${ }^{3}$ \\ Pawel Borowicz, ${ }^{4}$ Robert S. Brueggeman, ${ }^{2,3}$ and Paul Schwarz ${ }^{1, \dagger}$ \\ ${ }^{1}$ Department of Plant Sciences, North Dakota State University, Fargo, ND 58108, U.S.A. \\ ${ }^{2}$ Department of Crop and Soil Sciences, Washington State University, Pullman, WA 99164, U.S.A. \\ ${ }^{3}$ Department of Plant Pathology, North Dakota State University, Fargo, ND 58108, U.S.A. \\ ${ }^{4}$ Department of Animal Sciences, North Dakota State University, Fargo, ND 58108, U.S.A.
}

Accepted 11 March 2021.

Fusarium head blight (FHB) and the occurrence of mycotoxins is the largest food safety threat to malting and brewing grains. Worldwide surveys of commercial beers have reported that the trichothecene mycotoxin deoxynivalenol (DON) is the most frequent contaminant in beer. Although the DON content of grain generally declines during steeping due to its solubilization, Fusarium spp. can continue to grow and produce DON from steeping through the early kilning stage of malting. DON present on malt is largely extracted into beer. The objective of the current study was to localize the growth of Fusarium spp. within FHB-infected kernels by developing an improved method and to associate fungal growth with the production of DON during malting. FHBinfected barley, wheat, rye, and triticale grains that exhibited large increases in the amount of Fusarium Tri5 DNA and trichothecene mycotoxins following malting were screened for hyphal localization. The growth of fungal hyphae associated with grain and malt was imaged by scanning electron microscopy and confocal laser-scanning microscopy assisted with WGA-Alexa Fluor 488 staining, respectively. In barley, hyphae were present on or within the husk, vascular bundle, and pericarp cavities. Following malting, vast hyphal growth was observed not only in these regions but also in the aleurone layer, endosperm, and embryo. Extensive fungal growth was also observed following malting of wheat, rye, and

\section{${ }^{\dagger}$ Corresponding authors: S. Solanki; shyam.solanki@wsu.edu and} P. Schwarz; paul.schwarz@ndsu.edu

Funding: This material is based upon work supported by the United States Department of Agriculture (USDA) in a cooperative project with the U.S. Wheat \& Barley Scab Initiative under agreement number 590206-9-064, the National Science Foundation under grant number 0619098, and the American Society of Brewing Chemists (ASBC Research Council). Any opinions, findings, conclusions, or recommendations expressed in this publication are those of the author(s) and do not necessarily reflect the view of the USDA, National Science Foundation, or ASBC.

*The $e$-Xtra logo stands for "electronic extra" and indicates that a supplementary table and supplementary figures are published online.

The author(s) declare no conflict of interest. distributed under the CC BY 4.0 International license. triticale. However, these grains already had an extensive internal presence of Fusarium hyphae in the unmalted grain, thus representing an enhanced chance of fungal expansion during the malting.

Keywords: fluorescence, Fusarium head blight, hyphae, malting, microscopy, mycotoxins

The occurrence of mycotoxins is the largest food safety threat to raw materials in the malting and brewing industries, and recent worldwide surveys of commercial beers have shown deoxynivalenol (DON) to be a frequent, albeit low-level, contaminant (Kostelanska et al. 2009; Peters et al. 2017; Varga et al. 2013; Vidal et al. 2016; Wu et al. 2017). The source of mycotoxins in beer is the brewing grains, including malt and cereal adjuncts (Bauer et al. 2016; Scott 1996). In several cases, wheat beers have been reported to have higher DON levels than conventional barley-based beers. The production of trichothecenes, including DON, on small grains is associated with Fusarium head blight (FHB) or scab. In North America, infection is primarily caused by Fusarium graminearum, although other species are also reported to cause the disease (Jones and Mirocha 1999; Puri and Zhong 2010; Stack 1999). Several studies have shown that the DON present in malt is largely extracted into the resultant beer (Lancova et al. 2008; Schwarz et al. 1995). However, because the conditions of germination in malting provide a favorable environment for fungal growth and the production of DON and other mycotoxins, the malting process itself is of concern. The behavior of Fusarium spp. and the fate of DON during the malting of barley has been extensively investigated over the past two decades and were recently reviewed by Schwarz (2017). Results have generally suggested that grains with lower DON levels could often be used in malting because most of the DON present on barley was lost during the cleaning and steeping steps. It has also been observed that the viability of Fusarium spp. on barley decreases with time in storage. As such, problems with Fusarium growth and production of additional DON during the germination phase can often be reduced, if not eliminated, when infected grain with low DON is not malted until several months after harvest.

In the United States, self-imposed DON limits, ranging from $\leq 0.5$ to $1.0 \mathrm{mg} / \mathrm{kg}$, have been enacted by the industry. However, maltsters occasionally observe samples that exhibit aberrant behavior, where DON levels increase significantly during 
malting even after the barley has been stored for long periods. Thus, we hypothesize that the increased DON production during malting of these aberrant samples was also a result of internal Fusarium infection, as opposed to the typically reported external infection only. In support of this was the observation that pearling (abrasive milling for removal of outer tissues) of samples exhibiting this behavior did not result in the complete removal of DON, which was indicated by the commercial maltsters (personal communication). Recent work by Jin and coworkers (2018a, b) somewhat supports this hypothesis of early infection causing internal infection of Fusarium spp. in grains. Their investigations showed dramatic increases in Fusarium biomass and DON levels following the malting of 137 FHB-infected wheat, rye, and triticale samples. Although $75 \%$ of the initial grain samples had DON levels below the Food and Drug Administration advisory level of $1.0 \mathrm{mg} / \mathrm{kg}$, over $80 \%$ of the resultant malts had DON in excess of $1.0 \mathrm{mg} / \mathrm{kg}$. The growth of toxigenic Fusarium spp. was measured by quantifying Tri5 DNA in grains and malts, and levels exhibited a moderately strong relationship with malt DON (Vegi et al. 2011). An external versus internal infection may have some basis in fact because barley spikes are normally enclosed in the flag leaf sheath during anthesis (Alqudah and Schnurbusch 2017; Langevin et al. 2004), and exposure to the pathogen would occur only after the emergence of spikes. In contrast, in wheat, rye, and triticale, anthers are fully extruded at flowering, which occurs after spike emergence. In addition, rye is open pollinated and flowers for an extended period of time (Bonnett 1966). Exposure to the pathogen is earlier for these grains and could result in more extensive colonization within grains, when compared with the later infection of barley.

Growth of Fusarium spp. in grains during malting is, in a sense, an extension of the infection and colonization that have occurred in the field. Previous studies suggested that the Fusarium infection on grains was linked to the host anthesis and infection timing and patterns (Prom et al. 1999; Strange and Smith 1971; Trail 2009). During wheat anthesis, infection occurs when ascospores or macroconidia of $F$. graminearum deposit on or inside flowering spikelets. Hyphae then penetrate the ovary and eventually infect the floral bracts, including the paleae, lemma, and glume, through stomates. Wheat spikes are susceptible to infection from anthesis through the soft dough stage, and the fungus spreads through the rachis (Pritsch et al. 2000; Wanjiru et al. 2002). However, in barley, where spikes are still enclosed in the flag leaf sheath during anthesis, it has been reported that fungal growth occurs across the surface tissue of florets, prior to entry into natural cervices between the paleae and lemma (Lewandowski et al. 2006). Interaction of the pathogen with surface tissues of the barley grain and the differences in heading and flowering times have suggested that florets and their morphological characteristics potentially were determinants of the fungal infection and colonization process (Alqudah and Schnurbusch 2017; Imboden et al. 2018). Imboden et al. (2018) found that $F$. graminearum infected barley mainly through prickle-type trichomes on the outer surface of the paleae by trapping conidia and providing fungal penetration sites, compared with the dome-type trichomes that lack this function. In addition, floret maturity of barley influenced the disease spread on grain kernels because it was found that infections of more mature florets supported the spread of hyphae into the vascular bundle, whereas younger florets did not show this spread. The accumulation of silica in response to the presence of fungi on the paleae suggested a role for silica in pathogen establishment. Fusarium infection occurring largely on surface tissue of barley florets was supported by the work of Clear et al. (1997), who found that dehulling or pearling of hulless barley grains removed over $90 \%$ of the Fusarium spp. and about $50 \%$ of DON. However, pearling, which is an abrasive method, is not effective for the removal of pericarp tissue within the furrow creases regions.

In scanning electron microscopy (SEM) images of wheat kernels, Laca et al. (2006) showed that the distribution of mesophilic microorganisms, including bacteria and molds, was mainly localized in the grain coat tissues, including pericarp and the protein-rich layer below the pericarp. Jackowiak and coworkers (2005) (Packa et al. 2008) examined wheat and triticale inoculated with $F$. culmorum to show that the fungal hyphae were mainly observed in the areas of furrow creases, the brush end, the caryopsis coat, air spaces at the base of furrow creases, and the cavity located in the central endosperm. These central cavities are formed from repeated nuclear division during the differentiation of cell walls in the developing endosperm (Burton and Fincher 2014). Heneen and Brismar (1987) also reported microorganisms sometimes were found in the pericarp cavities and central endosperm cavities of shriveled triticale grains. Aside from SEM, fluorescence microscopy has been combined with fungal staining techniques to achieve the visualization of hyphae in host plant tissues, such as leaves and seedlings (Duckett and Read 1991; Hoch et al. 2005; Knight and Sutherland 2011; Moldenhauer et al. 2008). Recently, Solanki et al. (2019) developed a method of in planta fungal hyphae visualization using confocal laser-scanning microscopy (CLSM) and three-dimensional (3D) fungal hyphae reconstruction by Imaris software. This method achieves the highest standard of sample integrity by performing all of the WGA-Alexa Fluor 488 fluorescent dye poststaining steps on a single glass slide, thus eliminating the sample disturbance due to its movement between different vessels during the staining process, as required in previously reported methods (Ayliffe et al. 2011, Dugyala et al. 2015). The method developed by Solanki et al. (2019) is very efficient, cost effective, and rapid, and has high throughput. As such, it was well suited for the large number of samples needed in the current study.

We examined barley, wheat, rye, and triticale kernels for fungal growth and behavior during the malting process. The presence of $F$. graminearum as a predominant fungal species in the infected grains was confirmed by a shotgun sequencing approach. These samples were further analyzed by CLSM to observe the growth pattern of fungal hyphae prospering in the kernel tissues, employing a fluorescent staining method previously used by Solanki et al. (2019), with modifications in the sample preparation. These samples were also analyzed by SEM as an additional layer of visualization to complement the CLSM experiment. We found the presence of fungal hyphae within the FHB-infected small grains and the subsequent increase of fungal mass during the malting process. The quantitative PCR (qPCR) and gas chromatography confirmed the increased amount of Fusarium Tri5 DNA and type B trichothecene mycotoxins, respectively, in barley, wheat, rye, and triticale kernels subjected to malting. We concluded that the internal infestation of FHB hyphae in small grains survives the harsh treatment of pearling and malting and, upon favorable conditions, the subsequent increase in fungal mass contributed to the increased mycotoxin production during the malting process.

\section{RESULTS}

Fusarium presence, growth, and trichothecene production during malting.

Visual evidence of fungal infection was observed in most of the malt samples. For example, although the rye grains appeared clean (Supplementary Fig. S1A), a large number of pinkish kernels were visible following the malting process (Supplementary Fig. S1B). A variable pinkish color with white cottony surface growth is a visual phenotype used by pathologists to detect the 
presence of Fusarium spp. on the kernels. Pinkish kernels and cottony fungal surface growth were also seen in the naturally infected grains of barley, wheat, rye, and triticale and the resultant malts (Supplementary Fig. S2). However, the degree of these phenotypes varied among the samples. The kernels selected from FHB-infected wheat, rye, and triticale samples had some whitish discoloration or were shrunken yet the appearance of the infected barley was not much different than that of the control (Supplementary Fig. S2). Malt kernels resulting from FHB-infected grains displayed contrasting pink color. To ensure that the predominant fungus was a Fusarium sp. in the FHB grain and malt kernels, we used a shotgun sequencing approach based on our in-house Ion Torrent sequencing facility using a bulk sample approach for each type of sample. Our goal was only to experimentally validate the obvious presence of Fusarium spp. to complement our microscopy and trichothecene type B abundance study. Thus, we used a single sequencing profile for each of the five kernels and their malts and analyzed data for the abundance of Fusarium-specific sequencing reads (Supplementary Table S1).

A significant increase of Fusarium-specific sequencing reads was found in the malts of all grains when compared with the corresponding FHB-infected kernels $(P<0.05)$ (Supplementary Table S1). We also quantified a large amount of trichothecene production during the malting process accompanied by dramatic increases in Fusarium Tri5 DNA levels (Fig. 1) by employing gas-chromatography and qPCR, respectively. DON levels increased from 0.54 to $5.36 \mathrm{mg} / \mathrm{kg}$ for the malted barley, from $<0.20$ to $2.13 \mathrm{mg} / \mathrm{kg}$ for the malted wheat, from 0.45 to 9.35 $\mathrm{mg} / \mathrm{kg}$ for the malted rye, and from 0.86 to $23.81 \mathrm{mg} / \mathrm{kg}$ for the malted triticale (Fig. 1). Although contents of 3-acetyl-deoxynivalenol (3-ADON) and 15-acetyl-deoxynivalenol (15-ADON) were below $0.50 \mathrm{mg} / \mathrm{kg}$ in all unmalted grain samples, they were produced during malting. The content of 3-ADON increased to above $1.0 \mathrm{mg} / \mathrm{kg}$ in the malted triticale and barley and $15-\mathrm{ADON}$ to above $1.0 \mathrm{mg} / \mathrm{kg}$ in the malted triticale. The level of Tri5 DNA increased by 12.0-, 11.9-, 15.2-, and 18.7fold in the malted barley, wheat, rye, and triticale, respectively (Fig. 1). Levels of Fusarium Tri5 DNA and type B trichothecenes were considerably higher in the infected samples but were not detected in the control samples of grains and malt. Lower Tri5 DNA levels in malted barley and wheat samples likely

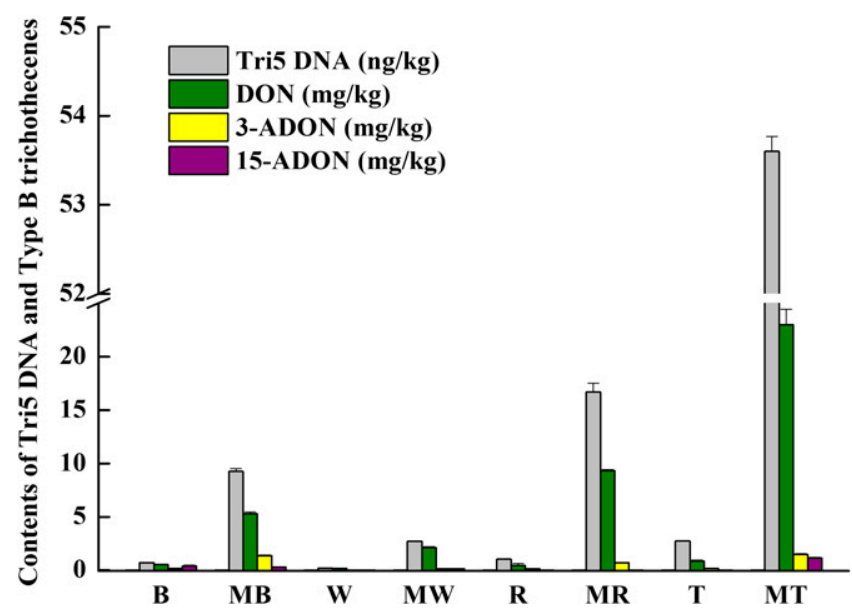

Fig. 1. Growth of Fusarium and trichothecene production in the malting of barley, wheat, rye, and triticale. The content of Tri5 DNA represents the biomass of Fusarium, and the detected trichothecenes include deoxynivalenol (DON), 3-acetyl-deoxynivalenol (3-ADON), and 15-acetyldeoxynivalenol (15-ADON). $\mathrm{B}=$ barley, $\mathrm{MB}=$ malted barley, $\mathrm{W}=$ wheat, $\mathrm{MW}=$ malted wheat, $\mathrm{R}=$ rye, $\mathrm{MR}=$ malted rye, $\mathrm{T}=$ triticale, and $\mathrm{MT}=$ malted triticale. reflected the use of kilned malt, as opposed to the malted rye and triticale, where freeze-dried samples were used. The kilned malt samples were derooted, which removes some Fusarium spp. and DON along with the rootlets. In addition, a small portion of DNA might be denatured by the high temperature of kilning.

\section{Growth patterns of fungal hyphae in Fusarium-infected} barley, wheat, rye, and triticale grain and malt kernels.

Samples were observed under the microscope to identify how the growth of Fusarium spp. that contributed to the increased Tri5 DNA and type B trichothecenes was spatially distributed. The FHB-infected barley samples were imaged with both SEM (Fig. 2A to D) and CLSM (Fig. 2E to H). On SEM, we found that the visual fungal infection was associated with the husk and furrow crease regions, as shown in the transverse section of the kernel (Fig. 2A). Fungal hyphae were seen on the surface of the husk (Fig. 2B), in the crevices under the husk (Fig. 2C), and in the pericarp cavity of the barley kernel (Fig. 2D). To better distinguish the internal growth pattern of fungal growth, we used fluorescently poststained samples on CLSM. During the imaging process, a green color was assigned to WGA-Alexa-fluor-488stained fungal hypha, and autofluorescence from grains was adjusted using a red channel using parameters defined elsewhere (Fig. 2E) (Solanki et al. 2019). We observed hyphae in the spongy parenchyma of the husk located on the furrow entrance (Fig. 2F), within the dorsal vein of husk (Fig. 2G), and in the vascular bundle (Fig. 2H). To our interest, there were even traces of hyphae localized in the tissues between the testa and aleurone layer (Fig. 2G), and in the nucellar projection transfer layer (NPTL) (Fig. 2H). However, no trace of fungal hyphae was found in the aleurone layer, starchy endosperm, and nucellar projection. With malted barley samples (Fig. 2I to P), we noticed an increased distribution of hyphae compared with unmalted barley kernels in the SEM and CLSM images (Fig. $2 \mathrm{~A}$ to $\mathrm{H}$ ). The transverse section of a malted barley kernel (Fig. 2I) and its subsequent high-resolution images (Fig. 2J to P) shows the extensive growth of hyphae filling the furrow crease regions (Fig. 2J) and crevices within the embryo (Fig. $2 \mathrm{~K}$ and L) of the malted kernel. Large amounts of hyphal penetration through grain tissues were observed within the husk, pericarp, testa, aleurone layer, and even the endosperm (Fig. 2M) in the CLSM images of fluorescently stained transverse sections. Hyphae had penetrated through the endosperm transfer layer (ETL) and NPTL (Fig. 2N), which refers to layers structurally surrounding the cavity. These layers were reported to be responsible for nutrient transfer from maternal to filial tissues and, as such, represent an important role in nourishment and solute supply for endosperm growth (Zheng and Wang 2011). The infection in transfer layers (Fig. 2N) was also observed to spread down into the starchy endosperm (Fig. 2O) and aside to the endosperm (Fig. 2P) when the conterminal locations of Figure $2 \mathrm{~N}, 2 \mathrm{O}$, and $2 \mathrm{P}$ were viewed in Figure $2 \mathrm{I}$.

In the FHB-infected wheat transverse section (Fig. 3A and E), we showed the overall infection locations, which demonstrate the extensive presence of hyphae in unmalted kernels. With SEM, hyphae were observed on the surface of the pericarp (Fig. 3B), and also extensively within the furrow (Fig. 3C) and pericarp cavities (Fig. 3D). With CLSM, hyphae were found to penetrate internal tissues, including the pericarp, testa, aleurone layer, and starchy endosperm (Fig. 3F); vascular bundle and nucellar projection (Fig. 3G); and NPTL and ETL (Fig. 3H). When malted wheat with FHB infection was observed with SEM, there was extensive hyphal growth on the surface of the kernel pericarp (Fig. 3J), and within the space of cavities and a few empty spaces in the starchy endosperm, possibly created by starch depletion due to fungal feeding (Fig. $3 \mathrm{~K}$ and $\mathrm{L}$ ). 
Examination with CLSM showed heavy infection in the nucellar projection, NPTL, and ETL (Fig. 3N); in the transfer layers, endosperm cavity, and endosperm (Fig. 3O); and within the testa, aleurone layer, and endosperm (Fig. 3P).

Similarly, FHB-infected rye and triticale displayed large amounts of hyphae on or within the observed samples (Fig. 4A to P). With SEM, fungal hyphae were observed on the surface of the testa (from which the pericarp had been peeled off) (Fig. $4 \mathrm{~A}$ ), in the space at the base of furrow creases and pericarp cavities (Fig. 4B), between starch granules (Fig. 4C), and within the embryo (Fig. 4D) of FHB-infected rye. Fungal hyphae had penetrated the internal tissues of infected rye such as furrow crease tissues and vascular bundle (Fig. 4E and F); ETL and and endosperm (Fig. 4G); and pericarp, testa, aleurone layer, and starchy endosperm (Fig. 4H), as seen in the CLSM images.

The distribution of hyphae in triticale grain using SEM is shown in Figure 4I to L. Penetration of hyphae through the grain surface into the pericarp is visible in Figure 4I and J. Large amounts of hyphae are also observed in the furrow (Fig. 4K) and the pericarp cavity (Fig. 4L). The presence of hyphae in a transverse section of a triticale kernel under CLSM is shown in Figure 4M. Hyphae were observed in the pericarp, testa, aleurone layer, and endosperm (Fig. 4N); furrow creases and the nearby endosperm (Fig. 4O); and ETL, NPTL, and endosperm
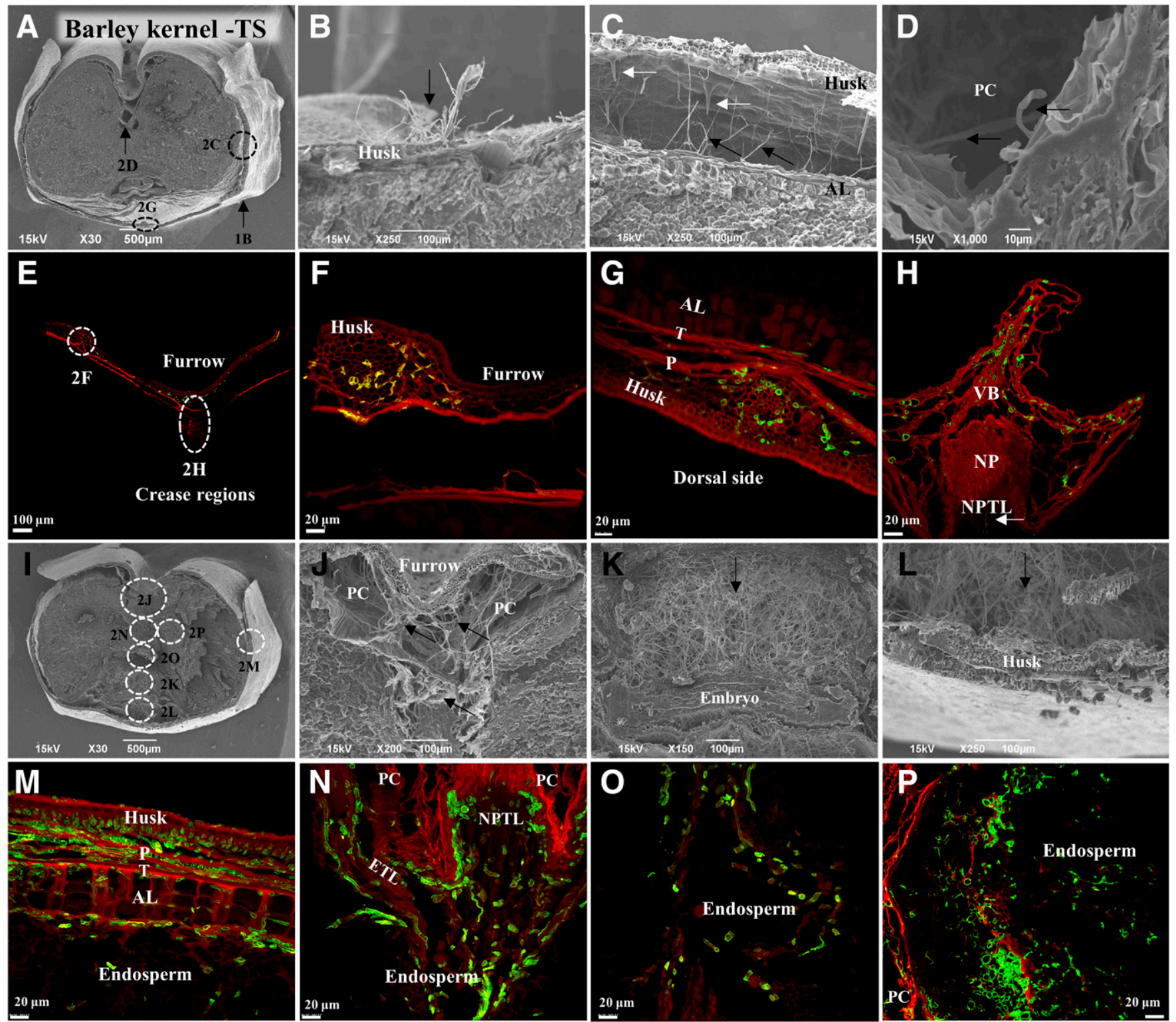

Fig. 2. Images of hyphae in the Fusarium head blight (FHB)-infected barley and malt kernels examined under scanning electron microscopy (SEM) and confocal laser-scanning microscopy (CLSM). A to $\mathbf{D}$ and $\mathbf{I}$ to $\mathbf{L}$, Imaged with SEM and $\mathbf{E}$ to $\mathbf{H}$ and $\mathbf{M}$ to $\mathbf{P}$, imaged with CLSM using WGA-Alexa Fluor 488 staining. In the CLSM images, the green color indicates fungal hyphae and the red color indicates host plant tissues. In the SEM images, hyphae were indicated with arrows in black. A to $\mathbf{H}$, Barley and I to P, malted barley. A, Transverse section of an FHB-infected barley kernel; B, hyphae on the husk surface; $\mathbf{C}$, hyphae under husk (white arrows indicate the prickle-type trichomes); $\mathbf{D}$, hyphae in a pericarp cavity (PC); $\mathbf{E}$, hyphae in the furrow crease (FC) regions; $\mathbf{F}$, hyphae within the spongy parenchyma of husk; $\mathbf{G}$, hyphae in husk, pericarp, and testa in the dorsal side; $\mathbf{H}$, hyphae within the furrow vascular bundle (VB) and nucellar projection (NP), with the arrow indicating a trace of hyphae under NP; $\mathbf{I}$, transverse section of FHB-infected barley malt; $\mathbf{J}$, hyphae in the space of PCs and FC crevices; $\mathbf{K}$ and $\mathbf{L}$, hyphae in the crevice of the embryo; $\mathbf{M}$, hyphae within husk, pericarp, testa, aleurone layer, and endosperm; $\mathbf{N}$, hyphae within the cavity-surrounding endosperm transfer layer and NP transfer layer; $\mathbf{O}$, hyphae within endosperm below panel $\mathbf{N}$; and $\mathbf{P}$, hyphae present within the endosperm at the left of the furrow. AL $=$ aleurone layer, ETL $=$ endosperm transfer layer, $\mathrm{NPTL}=$ nucellar projection transfer layer, $\mathrm{P}=$ pericarp, $\mathrm{T}=$ testa, and $\mathrm{TS}=$ transverse section. 
cavity (Fig. 4P). Localization of hyphae in FHB-infected rye and triticale malt was very similar to that observed in unmalted grains. However, the presence of hyphae tended to be much more extensive in malt kernels, suggesting growth during malting.

Control samples, representing grains with nondetectable DON and Tri5 DNA levels, were stained concurrently with those that were infected. Small amounts of hyphae were detected in tissues of these grains or malts (Fig. 5). In barley, some hyphae are visible mainly in the spongy parenchyma of the husk (Fig. 5A) and, in wheat, within the pericarp and sieve element located in the furrow region (Fig. 5B). Results were similar for rye, with hyphae being detected within the pericarp and testa (Fig. 5C) whereas, for triticale, hyphae were present in the seed coat and vascular bundle in the furrow region, and even within the ETL under nucellar projection (Fig. 5D).

\section{DISCUSSION}

For centuries, maltsters and brewers have relied on the visual appearance of grain and malt as an indicator of potential microbial problems, and pink grains have been considered to suggest infection with Fusarium spp. (Schwarz 2003). Empirically, symptoms such as reddish and black discoloration of kernels (Supplementary Figs. S1 and S2) can be related to Fusarium infection but this symptomatology has generally shown a low
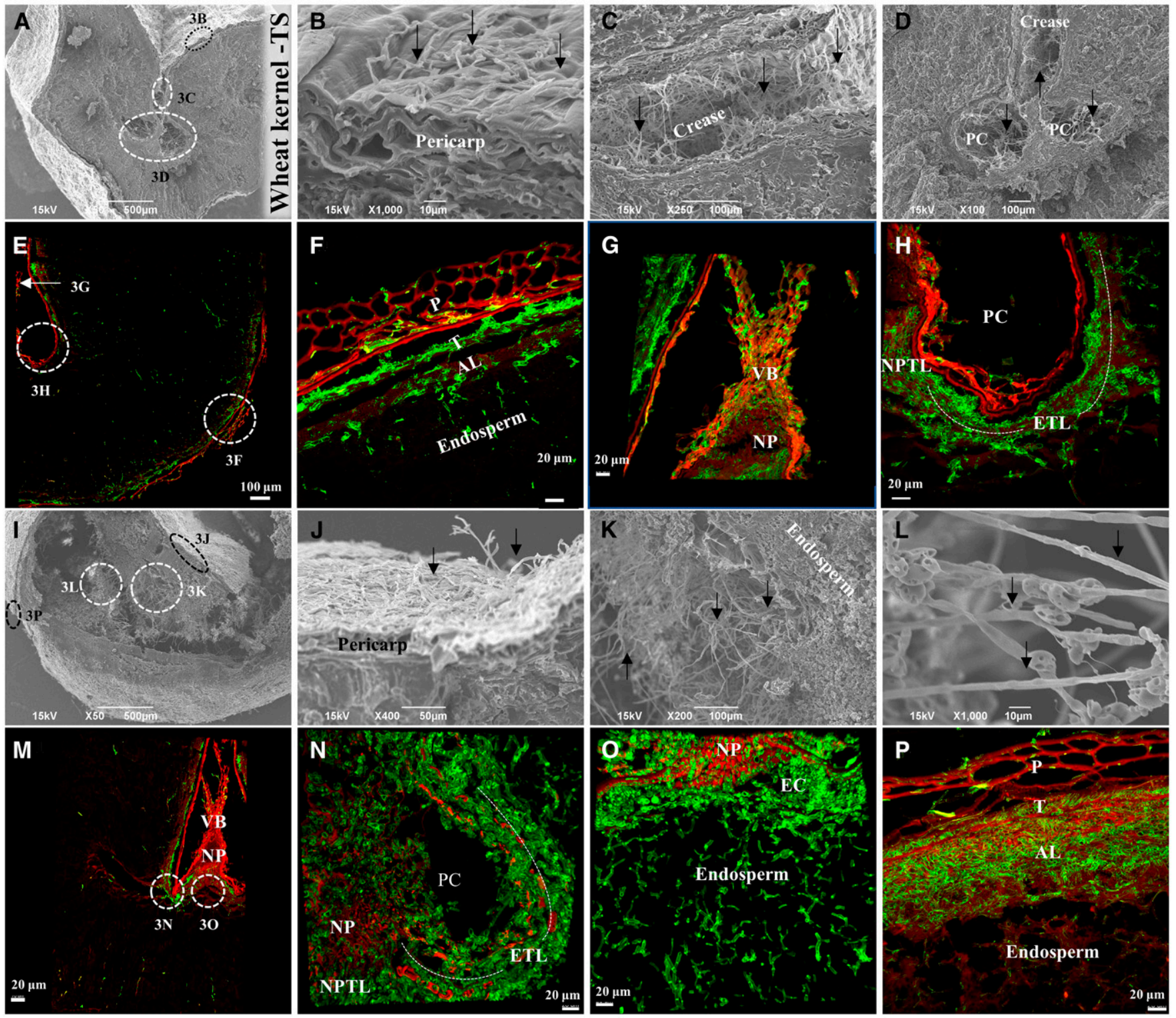

Fig. 3. Images of fungal hyphae in the Fusarium head blight (FHB)-infected wheat and malt kernels visualized with scanning electron microscopy (SEM) and confocal laser-scanning microscopy (CLSM). A to $\mathbf{D}$ and $\mathbf{I}$ to $\mathbf{L}$, Imaged with SEM and $\mathbf{E}$ to $\mathbf{H}$ and $\mathbf{M}$ to $\mathbf{P}$, imaged with CLSM using WGA-Alexa Fluor 488 prestaining. In the CLSM images, the green color indicates fungal hyphae and the red color indicates host plant tissues. In the SEM images, hyphae were indicated with arrows in black. A to $\mathbf{H}$, Wheat and $\mathbf{I}$ to $\mathbf{P}$, malted wheat. A, Transverse section of an FHB-infected wheat kernel; B, hyphae on the surface of pericarp; C, hyphae in the furrow creases (FCs); D, hyphae in the pericarp cavities (PCs); E, hyphae in a transverse section of an FHB-infected wheat kernel; F, hyphae within pericarp, testa, aleurone layer, and endosperm; G, hyphae within the vascular bundle (VB) and nucellar projection (NP); H, hyphae within the NP transfer layer and endosperm transfer layer; I, transverse section of an FHB-infected malted wheat kernel; $\mathbf{J}$, hyphae on the surface of pericarp; $\mathbf{K}$ and $\mathbf{L}$, hyphae within crevices of the endosperm; $\mathbf{M}$, transverse section of an FHB-infected malted wheat kernel; N, hyphae within the NP, endosperm transfer layer, and NP transfer layer; $\mathbf{O}$, hyphae within the transfer layers, endosperm cavity, and endosperm; and $\mathbf{P}$, hyphae within the pericarp, testa, aleurone layer, and starchy endosperm. AL $=$ aleurone layer, EC $=$ endosperm cavity, ETL $=$ endosperm transfer layer, NPTL $=$ nucellar projection transfer layer, $\mathrm{P}=$ pericarp, $\mathrm{T}=$ testa, and $\mathrm{TS}=$ transverse section. 
predictive value for Fusarium contamination because infection by other fungi such as Rhodotorula and Cryptococcus spp. can cause similar discoloration (Laitila et al. 2011). In the current study, we demonstrated that Fusarium spp. were the predominant fungi in the infected samples (Supplementary Table S1). We show that, during the malting process, there was an increased distribution of $F$. graminearum hyphae in the inner layers of barley, wheat, rye, and triticale kernels that was accompanied by increased levels of Tri5 DNA and DON. With barley, a small amount of Fusarium hyphae was present in the unmalted grains and mainly distributed in the husk and vascular bundle. However, F. graminearum increased to become the predominant fungal organism found in the interior of barley malt kernels (Supplementary Table S1). The contents of both Fusarium Tri5 DNA and DON increased more than 10 times following the malting of all of the grains, which further demonstrated the dramatic growth of Fusarium spp. during malting (Fig. 1). This behavior was different from a previous report that barley grains with low DON level (e.g., $<0.5 \mathrm{mg} / \mathrm{kg}$ ) generally yielded lower DON levels on the malt (Schwarz et al. 2006). Differences may be associated with the physical location of Fusarium spp. within kernels.

To achieve the objective of fungal hyphae growth tracking in the seed kernels, we adopted a method described by Solanki et al. (2019) and modified it for visualization of fungal growth in the transverse section of kernels. The original poststaining
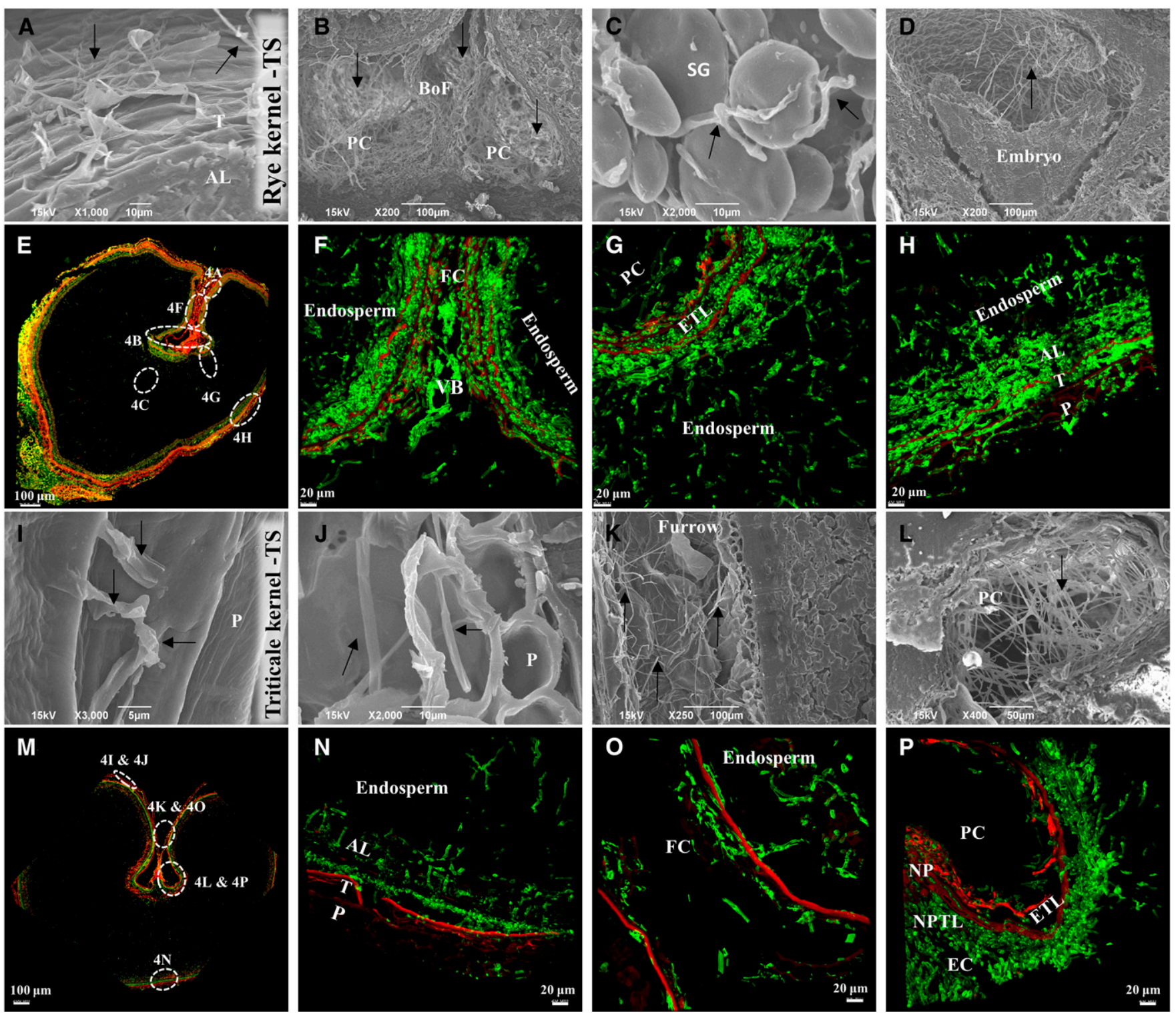

Fig. 4. Images of hyphae in Fusarium head blight (FHB)-infected rye and triticale kernels examined with scanning electron microscopy (SEM) and confocal laser-scanning microscopy (CLSM). A to $\mathbf{D}$ and $\mathbf{I}$ to $\mathbf{L}$, Imaged with SEM and $\mathbf{E}$ to $\mathbf{H}$ and $\mathbf{M}$ to $\mathbf{P}$, imaged with CLSM using WGA-Alexa Fluor 488 prestaining. In the CLSM images, the green color indicates fungal hyphae and the red color indicates host plant tissues. In the SEM images, hyphae were indicated with arrows in black. A to $\mathbf{H}$, Rye and $\mathbf{I}$ to $\mathbf{P}$, triticale. A, Hyphae on the surface of testa, with the pericarp peeled off; $\mathbf{B}$, hyphae in the air space at the base of furrow creases (FCs) and pericarp cavities (PCs); C, hyphae between starch granules; D, hyphae in the crevices of the embryo; $\mathbf{E}$, transverse section of an FHB-infected rye kernel; F, hyphae within the furrow creases, vascular bundle (VB), and nearby endosperm $\mathbf{G}$, hyphae within the endosperm transfer layer and endosperm; $\mathbf{H}$, hyphae within pericarp, testa, aleurone layer, and endosperm; $\mathbf{I}$, penetration of hyphae through the pericarp surface; J, hyphae in the pericarp; $\mathbf{K}$, hyphae in the air space of furrow; $\mathbf{L}$, hyphae in a PC, with the location indicated in panel M; M, transverse section of an FHB-infected triticale kernel; N, hyphae within pericarp, testa, aleurone layer, and endosperm; $\mathbf{O}$, hyphae within the FCs and the nearby endosperm; and $\mathbf{P}$, hyphae within the nucellar projection (NP), NP transfer layer, endosperm transfer layer, and endosperm cavity. AL $=$ aleurone layer, $\mathrm{BoF}=$ the base of furrow creases, $\mathrm{EC}=$ endosperm cavity, $\mathrm{ETL}=$ endosperm transfer layer, NPTL $=$ nucellar projection transfer layer, $\mathrm{P}=$ pericarp, $\mathrm{SG}=$ starch granule, and $\mathrm{T}=$ testa . 
method (Solanki et al. 2019) uses a rapid, high-throughput, lowcost sample preparation approach and retains the sample integrity due to processing of tissues on a single glass slide, thus avoiding the disturbance of samples in each step of traditional staining and washing. Due to the direct sample tissue staining approach on the glass slides with a hydrophobic boundary, this method minimizes the use of per-sample WGA-Alexa Fluor 488 fluorescent dye. Considering that we were interested in processing multiple types of kernel samples, with and without FHB, this method was very well suited for our purpose. However, there were some technical challenges when adopting the method for grain samples which contain large amounts of starch granules. Unlike relatively thin and flat plant leaves, the whole grain and malt kernels are roundish, thick, and nontransparent due to nonopaque starchy endosperm. Thus, kernels required sectioning into thin layers of 10 to $20 \mu \mathrm{m}$. Large portions of thin sections of malt kernels were often lost, particularly when the endosperm had been significantly modified by germination. Although Solanki et al. (2019) recommended the autoclaving of leaves in a liquid cycle to remove wax from leaf tissues, when applied to grain, autoclaving tended to cook the samples and made them too soft to section. As an alternative, we evaluated soaking in $10 \%$ neutral buffered formalin (NBF) fixative solution prior to the sectioning. We observed that the grain and malt kernels retained their integrity when soaked for $48 \mathrm{~h}$ before sectioning into 10 - to $15-\mu \mathrm{m}$ sections. These sections were thicker than the 4- to 5- $\mu \mathrm{m}$ sections of leaf tissue previously used for microscopic studies (Jääskeläinen et al. 2013; Olkku et al. 2005). In order to improve the permeability of fluorescent stain, we increased the staining time to $5 \mathrm{~h}$ and replenished the fluorescent solution after $2.5 \mathrm{~h}$ The Z-stack images scanned by CLSM had a desired intact structure of high resolution (Figs. 2, 3,4 , and 5). Small amounts of hyphae were detected even in the relatively clean grain kernels (Fig. 5), proving the sensitivity of this method to localize hyphae in grain and malt. This is important, because these hyphae might be inaccessible during cleaning and, thus, become potential sources of hyphal growth during malting.

Our image analysis showed that the "internal" infection of barley typically occurred in vascular bundles and cavities located in the furrow creases region (Fig. 2). Hyphae in these regions are likely protected from removal during grain cleaning and steeping and, thus, can thrive during the malting process. Imboden et al. (2018) observed that spread of inoculated F. graminearum on the furrow vascular bundle tended to occur in mature florets but not in younger ones. Timing of infection and maturity of florets could be possibly influenced by the weather and variety of barley (Prom et al. 1999). To localize the fungal hyphae within FHB-infected grain and malt samples, SEM and CLSM were both used because of their respective advantages. CLSM with the Z-Stack function was capable of imaging hyphae which had penetrated through host tissues. However, because of the extensive washing used in sample preparation, there was a high probability that less-adherent hyphae were removed. Although SEM was only used to examine the surface of an object, minimal sample preparation has the advantage of avoiding the loss of hyphae from host tissues. The complementary nature of the two techniques was illustrated by comparing the SEM and CLSM images of the same kernel locations. For instance, hyphae were obviously seen within the cavities of barley and malt (Fig. 2D and J) but were not visible in the surrounding host tissues when viewed under SEM. In contrast, when viewed using CLSM and prestaining, there was clearly a presence of hyphae within the barley host tissues (Fig. $2 \mathrm{H}$ and N). Complementary comparisons of the cavities for wheat (Fig. 3D and H), rye (Fig. 4B, F, and G), and triticale (Fig. 4L and P) further reflected the suitability of this approach. SEM examination showed hyphae on the surface of barley (Fig. 2B) and under the husk (Fig. 2C), and on the pericarp of wheat and malt (Fig. $3 \mathrm{~B}$ and $\mathrm{J}$ ), rye (Fig. 4A), and triticale (Fig. 4I). In addition, with CLSM, hyphae were observed within tissues, including spongy parenchyma of the husk (Fig. 2F, G, and M) and the pericarp of wheat (Fig. 3F and P), rye (Fig. 4H), and triticale (Fig. 4N). Previous studies using SEM confirmed the presence of hyphae on the kernel surface and within the cavities of FHB or shriveled wheat, rye, and triticale grains, and the authors speculated on possible infection in the endosperm (Heneen and Brismar 1987; Jackowiak et al. 2005; Packa et al. 2008). In the current study, hyphae were observed physically in crevices of vascular bundle under SEM and within the tissues and nearby endosperm under CLSM of malted barley (Fig. 2J versus $\mathrm{N}$ to P), wheat (Fig. 3C versus $G$ ), rye (Fig. $4 \mathrm{~B}$ versus $\mathrm{F}$ and $\mathrm{G}$ ), and triticale (Fig. $4 \mathrm{~K}$ versus $\mathrm{O}$ ).

In terms of external versus internal infection on kernels, the SEM and CLSM images were aligned to analyze the localization of hyphae on or within each of the grain and malt samples. Only slight infection was observed in the FHB-infected barley grain kernels, mostly in the husk and furrow regions of the husk. Hyphae were mainly localized on the husk surface (Fig. 2B), in areas under the husk (Fig. 2C), and in the spongy parenchyma and cementing layer of husk (Fig. $2 \mathrm{~F}$ and G). The distribution of hyphae in the husk likely resulted from the surface infection of Fusarium spp. on florets of barley in the field (Langevin et al. 2004), and it is worth noting that the prickle-type
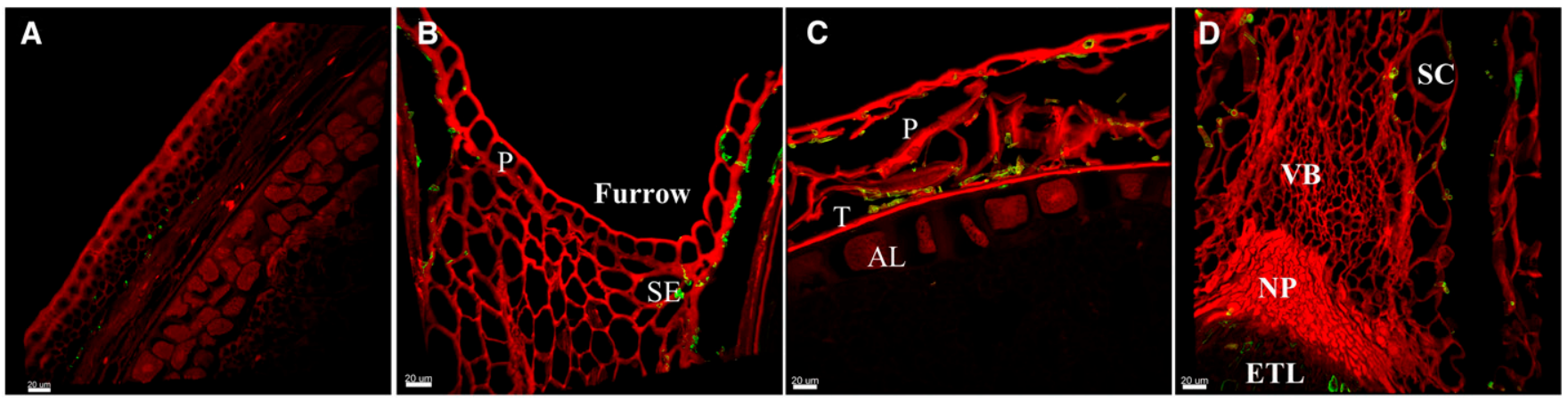

Fig. 5. Images of hyphae in control samples of noninfected or limited Fusarium-infected samples using confocal laser-scanning microscopy and prestaining with WGA-Alexa Fluor 488. A, Hyphae observed within the spongy parenchyma of barley husk; B, hyphae within pericarp, and sieve element located in the furrow region of wheat; $\mathbf{C}$, hyphae within the pericarp and testa of rye but not in the aleurone layer; and $\mathbf{D}$, hyphae within the furrow seed coat, vascular bundle, endosperm transfer layer, and under the nucellar projection of triticale. $\mathrm{AL}=$ aleurone layer, $\mathrm{P}=$ pericarp, $\mathrm{SE}=$ sieve element, $\mathrm{T}=$ testa, $\mathrm{SC}=$ seed coat, $\mathrm{VB}=$ vascular bundle, $\mathrm{ETL}=$ endosperm transfer layer, and $\mathrm{NP}=$ nucellar projection. 
trichomes, which have been suggested to have the function of trapping conidia and to act as sites of fungal penetration (Imboden et al. 2018), were observed on the inner surface of barley husk (Fig. 2C). Within the furrow, hyphae were present in the vascular bundle (Fig. $2 \mathrm{E}$ and $\mathrm{H}$ ) that were created by the fusion of margins of lemma with three layers and paleae with two layers of vascular bundle. Fungal infection at the furrow margin (Fig. 2F) was probably caused by the hyphal penetration through stomates that were observed to run along the vascular bundle ridges (Imboden et al. 2018). The furrows and the paleae margins were considered to be the frequent location of lesion initiation on grains grown in the field (Lewandowski et al. 2006). In addition, hyphae were observed in cavities under the vascular bundle (Fig. 2D) and dorsal vein (Fig. 2G). Although the infection mechanisms at these two sites were not clear, the inaccessibility of these locations during grain cleaning and steeping prior to germination suggests that they might be a source of the fungal growth during malting. In a previous study, Olkku et al. (2005) found that DNA stained with 4',6-diamidino-2-phenylindole (DAPI) was located in the pericarp and endosperm of barley and they speculated on the existence of microbes in these tissues. However, DAPI is not specific to microbial DNA. In the current study, extensive growth of hyphae was also observed at these tissues following malting of the barley. This resulted in not only the profuse growth of hyphae in the internal crevices such as cavities (Fig. 2J) and embryo regions (Fig. 2K and L) but also penetration of hyphae into host tissues (Fig. 2M to P). The presence of hyphae in the starchy endosperm was facilitated by spread through the husk, pericarp, testa, and aleurone layer (Fig. 2M) and, alternatively, through the ETL, which connected the pericarp cavity to endosperm (Fig. 2N and P). These results illustrated that even a trace amount of fungal infection in the interior of grains can potentially result in significant growth under favorable conditions of malting.

With FHB-infected wheat, rye, and triticale, extensive growth of hyphae was observed both on the kernel surface and within the interior of grains prior to malting (Figs. 3A to $\mathrm{H}$ and $4 \mathrm{~A}$ to $\mathrm{P}$ ), and fungal growth substantially increased following the malting (Fig. 3I to P). The growth of hyphae into grain tissues such as the aleurone layer and endosperm was not observed in the previous studies using SEM (Jackowiak et al. 2005; Packa et al. 2008). However, Jackowiak et al. (2005) and Packa et al. (2008) both reported that amylolytic enzymes produced by the Fusarium spp. used in inoculation were able to diffuse through the aleurone layer and endosperm, causing fractured cells in the aleurone layer and damaged starch granules, which were not even in contact with hyphae. The physical distribution of hyphae was associated with the timing of Fusarium infection on these hulless grains and the pattern of Fusarium infection in the field. Wheat, rye, and triticale flower after heading, and the primary infection occurs in the field when ascospores (sexual spores) or macroconidia (asexual spores) are released from soilborne debris and then infect flowering spikelets. Moreover, the infection spreads between florets through the rachis, which results in the fungus entering the base of caryopsis (Gaikpa et al. 2020; Langevin et al. 2004). These patterns of infection could lead to more extensive colonization of Fusarium spp. in the interior of wheat, rye, and triticale grain kernels, which provided potential sources of fungal growth and mycotoxin production during malting.

In summary, we investigated the physical localization of fungal hyphae in FHB-infected grains that had displayed large increases in DON following malting. In all cases, extensive internal fungal colonization was observed to occur during malting. Our data identify the fungal growth pattern in grain tissues and shed light on how the favorable conditions of the malting process can result in unchecked growth of Fusarium spp. and toxicity of the end product.

\section{MATERIALS AND METHODS}

\section{Collection of grain and malt samples.}

Grains and their corresponding malt samples were from a variety of sources. They were all selected on the basis that they had exhibited large increases in DON following malting, despite the original grain showing relatively low levels. Two-rowed spring barley (variety Copeland) was from the 2016 commercial crop in Saskatchewan, Canada. Both the barley and corresponding malts were provided by a commercial malting company which had identified this as a problematic sample in terms of DON production. The hard red spring wheat sample (variety Barlow) was from the 2015 commercial crop in North Dakota, and had been micromalted in a previous study (Jin et al. 2018b). Rye (variety Hazlet) and triticale (variety Tulus) samples were from University of Minnesota field trials in 2016. Micromalts of these grains were from a previous study (Jin et al. 2018a). Noninfected or slightly FHB-infected barley, wheat, rye, and triticale samples were identified by screening for Tri5 DNA and DON and used as negative controls for microscopic examination.

\section{qPCR quantification of Fusarium Tri5 DNA in FHB and non-FHB grains and malt.}

Fusarium Tri5 DNA was quantitatively analyzed by $\mathrm{qPCR}$ as described by Jin et al. (2018a). The fungal DNA was extracted with a DNeasy Plant Mini Kit (Qiagen Inc. Valencia, CA, U.S.A.), amplified with Fusarium Tri5 DNA PCR primers TMT_fw (5'-GATTGAGCAGTACAACTTTGG-3') and TMT_rev (5'-ACCATCCAGTTCTCCATCTG-3') (Milliporesigma, Burlington, MA, U.S.A.), and quantified with SsoAdvanced Universal SYBR Green Supermix (Bio-Rad, Hercules, CA, U.S.A.). To quantify the initial amount of Tri5 DNA for each sample, the qPCR assays were performed simultaneously with a serial dilution of purified $F$. graminearum Tri5 PCR amplicon generated with $F$. graminearum DNA template and extracted from single-spore cultures. The logarithm of odds (LOD) and limit of quantitation (LOQ) of Tri5 DNA level were 0.10 and $1.0 \mathrm{ng} / \mathrm{kg}$, respectively.

\section{Quantification of type B trichothecenes in FHB and non-FHB grains and malt.}

DON, 3-ADON, 15-ADON, and nivalenol (NIV) were determined according to our standard laboratory procedure (Jin et al. 2018b; Wan et al. 2018). Ground samples $(2.50 \mathrm{~g})$ were extracted with $20 \mathrm{ml}$ of acetonitrile/water (84:16, vol/vol) for 1 h. Extracts $(4.0 \mathrm{ml})$ were filtered through a C18/alumina (1:3, $\mathrm{wt} / \mathrm{wt})$ column, and an aliquot $(2.0 \mathrm{ml})$ of the supernatant was dried under compressed air at $50^{\circ} \mathrm{C}$ for an hour. The dried samples were derivatized with $100 \mu \mathrm{l}$ of BSA/TMCS/TMSI (3:2:3, $\mathrm{vol} / \mathrm{vol} / \mathrm{vol}$ ). An aliquot of $1.0 \mathrm{ml}$ of isooctane consisting of Mirex $(0.5 \mu \mathrm{g} / \mathrm{ml})$ as an internal standard was added into the derivatized sample; then, the derivatization was terminated by adding $1.0 \mathrm{ml}$ of $\mathrm{NaHCO}_{3}(3 \%, \mathrm{wt} / \mathrm{vol})$ solution. The derivated mycotoxins extracted in the isooctane layer were analyzed on an Agilent $6890 \mathrm{~N}$ gas chromatograph with a 5973 Mass Selective Detector (Agilent Technologies, Santa Clara, CA, U.S.A.) under SIM mode. The separation was performed on a $35 \%$ phenyl siloxane column ( $30.0 \mathrm{~m}$ by $250 \mu \mathrm{m}, 0.25 \mu \mathrm{m}$ nominal) (Agilent HP-35, part number 19091G-133; Agilent Technologies) as previously described. The ions of $295.20,422.20,512.30$, and 497.30 were used for the DON derivative qualification; 377.20 , $392.20,467.20$, and 482.30 for $3-A D O N ; 392.20,407.20$, 467.20, and 482.20 for 15-ADON; 379.20, 482.30, 510.30, and 585.30 for NIV; and 271.90, 236.90, 331.90, and 403.70 for Mirex. The ion of 271.90 was used for the quantification of Mirex, and the areas of 295.20, 377.20, 392.20, and 271.90 were used to make the standard curves for quantifying DON, 
3-ADON, 15-ADON, and NIV, respectively. The LOD and LOQ for these type B trichothecenes were 0.10 and $0.20 \mathrm{mg} / \mathrm{kg}$, respectively.

\section{Ion Torrent sequencing and data analysis to identify Fusarium spp. in FHB and non-FHB grains and malt.}

We applied our in-house shotgun sequencing approach to confirm the presence of Fusarium spp. in the FHB-infected and clean kernels. Because our specific objective was to complement our microscopy efforts and to identify whether the predominant fungal species was Fusarium, we only used a single sequencing profile for each of the five kernels and their malts. In terms of experimental design, two treatments consisted of sample types: (i) FHB-infected grain and (ii) the corresponding malts. Each sample consisted of subsamples of wheat, barley, rye, and triticale kernels, thus allowing us to run a $t$ test to determine whether a significant increase of Fusarium spp. occurred upon malting of kernels. However, due to having only one replicate of each subsample type, any further analysis such as a descriptive metagenome identification cannot be correctly reported due to the lack of statistical power; however, we have submitted Supplementary Table S1 with identified additional species in the test samples to provide a rough estimate. However, a metagenome description was not provided in the article due to abovementioned reasons. To specifically identify the internal microorganisms in the kernels, husk and pericarp tissues were removed from selected kernels. For decoating, $1.0 \mathrm{~g}$ of each grain or malt sample was washed with $1 \%$ sodium hypochlorite, then soaked in $75 \%$ ethanol for $12 \mathrm{~h}$. The husk of barley and malt kernels and the pericarp of wheat, rye, triticale, and the malt kernels were loosened by gently rubbing them in a mortar and pestle. Kernels were then peeled with a tweezer to remove the husk or pericarp. The dehusked and decoated kernels were washed with sterile water. A DNeasy Plant Mini Kit (Qiagen Inc.) was used for the total DNA extraction from four to five seeds. DNA samples were then utilized to generate a wholegenome shotgun metagenomic sequencing library and sequenced in an in-house Ion Torrent S5 platform. Barcoded samplespecific sublibraries were prepared using the NEBNext Fast DNA Fragmentation and Library Prep Set for Ion Torrent kit. The sequencing data were used to perform a blastX search in the regularly updated in-house NCBI nonredundant (NCBI-nr) database utilizing the high-throughput alignment program DIAMOND (Buchfink et al. 2015). The microbiome analysis tool MEGAN Community Edition v6.12.3 (Huson et al. 2016) was used to analyze the blastX alignment results generated in DIAMOND and assign individual reads to operational taxonomic units, and Fusarium reads were extracted from the captured species (Supplementary Table S1). Considering that our aim was to identify the abundance of Fusarium spp., we limited our analysis and submitted the raw and processed files for community use. A $t$ test comparing infected versus noninfected samples for percentage of Fusarium reads (Supplementary Table S1) was carried out in the R (4.1) software platform. The raw sequencing files were submitted in the NCBI Sequence Read Archive database (PRJNA647973)

\section{CLSM examination of cereal grains and malt.}

Ten kernels of each sample of grain and malt were soaked in $10 \% \mathrm{NBF}$ solution for 12,24 , or $48 \mathrm{~h}$ at room temperature to fix the kernels for ease for sectioning. Fixed kernels were dehydrated in a series of alcohol washes; then, two to five kernels were embedded in a paraffin wax block. Transverse sections (10 $\mu \mathrm{m}$ in thickness) were taken from the middle portion of kernels within the paraffin block on a manual microtome (Leica, Heidelberg, Germany). Sections were collected on Superfrost plus charged slides (Thermo Fisher Scientific, Waltham, MA,
U.S.A.) for better attachment (Solanki et al. 2020). Two to three such combined sections were made for each sample. These sample sections were stained with WGA-Alexa Fluor 488 (Invitrogen, Eugene, OR, U.S.A.) at $20 \mu \mathrm{g} / \mathrm{ml}$ as described by Solanki et al. (2019). However, the staining time was optimized and extended to $5 \mathrm{~h}$ without shaking for better penetration of stain. The staining solution was replenished after $2.5 \mathrm{~h}$ to maintain the strength of fluorescent activity. Prepared slides were visualized on an LSM 700 laser microscope (Zeiss, Oberkochen, Germany) under a $\times 40$ magnification oil immersion lens and $\times 10$ objective lens using two different fluorescence channels. Green channel 488 was assigned for WGA-Alexa Fluor staining and red channel 555 was used for plant tissues with autofluorescence detection. To visualize inner structures in the section, Z-stack images were obtained (20 to 100 images per infection site) and were processed on Imaris 9.0.1 software for the 3D visualization of the fungal infection in the samples (Zeiss).

\section{SEM analysis of grain and malt kernels.}

Grain and malt kernels were cut in half transversely with a razor blade to expose the interior and attached to cylindrical aluminum mounts with colloidal silver paint (SPI Supplies, West Chester, PA, U.S.A.). Then, they were sputter coated (Cressington 108auto; Ted Pella, Redding, CA U.S.A.) with a conductive layer of gold. Images were obtained with a JEOL JSM-6490LV scanning electron microscope (JEOL USA, Inc., Peabody, MAs U.S.A.) at an accelerating voltage of $15 \mathrm{kV}$.

\section{LITERATURE CITED}

Alqudah, A. M., and Schnurbusch, T. 2017. Heading date is not flowering time in spring barley. Front. Plant Sci. 8:896.

Ayliffe, M., Devilla, R., Mago, R., White, R., Talbot, M., Pryor, A., and Leung, H. 2011. Nonhost resistance of rice to rust pathogens. Mol. Plant-Microbe Interact. 24:1143-1155.

Bauer, J. I., Gross, M., Gottschalk, C., and Usleber, E. 2016. Investigations on the occurrence of mycotoxins in beer. Food Control 63:135139.

Bonnett, O. T. 1966. Inflorescences of maize, wheat, rye, barley, and oats: Their initiation and development. Univ. Ill. Agric. Exp. Stn. Bull. University of Illinois (Urbana-Champaign campus), Urbana, IL, U.S.A.

Buchfink, B., Xie, C., and Huson, D. H. 2015. Fast and sensitive protein alignment using DIAMOND. Nat. Methods 12:59-60.

Burton, R. A., and Fincher, G. B. 2014. Evolution and development of cell walls in cereal grains. Front. Plant Sci. 5:456.

Clear, R., Patrick, S., Nowicki, T., Gaba, D., Edney, M., and Babb, J. C. 1997. The effect of hull removal and pearling on Fusarium species and trichothecenes in hulless barley. Can. J. Plant Sci. 77:161-166.

Duckett, J. G., and Read, D. J. 1991. The use of the fluorescent dye, for selective staining of ascomycete fungi associated with liverwort rhizoids and ericoid mycorrhizal roots. New Phytol. 118:259-272.

Dugyala, S., Borowicz, P., and Acevedo, M. 2015. Rapid protocol for visualization of rust fungi structures using fluorochrome Uvitex $2 \mathrm{~B}$. Plant Methods 11:54.

Gaikpa, D. S., Lieberherr, B., Maurer, H. P., Longin, C. F. H., and Miedaner, T. 2020. Comparison of rye, triticale, durum wheat and bread wheat genotypes for Fusarium head blight resistance and deoxynivalenol contamination. Plant Breed. 139:251-262.

Heneen, W. K., and Brismar, K. 1987. Scanning electron microscopy of mature grains of rye, wheat and triticale with emphasis on grain shrivelling. Hereditas 107:147-162.

Hoch, H. C., Galvani, C. D., Szarowski, D. H., and Turner, J. N. 2005. Two new fluorescent dyes applicable for visualization of fungal cell walls. Mycologia 97:580-588.

Huson, D. H., Beier, S., Flade, I., Górska, A., El-Hadidi, M., Mitra, S. Ruscheweyh, H. J., and Tappu, R. 2016. MEGAN Community Edition-Interactive exploration and analysis of large-scale microbiome sequencing data. PLOS Comput. Biol. 12:e1004957.

Imboden, L., Afton, D., and Trail, F. 2018. Surface interactions of Fusarium graminearum on barley. Mol. Plant Pathol. 19:1332-1342. 
Jääskeläinen, A., Holopainen-Mantila, U., Tamminen, T., and Vuorinen, T. 2013. Endosperm and aleurone cell structure in barley and wheat as studied by optical and Raman microscopy. J. Cereal Sci. 57:543-550.

Jackowiak, H., Packa, D., Wiwart, M., and Perkowski, J. 2005. Scanning electron microscopy of Fusarium damaged kernels of spring wheat. Int. J. Food Microbiol. 98:113-123.

Jin, Z., Gillespie, J., Barr, J., Wiersma, J. J., Sorrells, M. E., Zwinger, S., Gross, T., Cumming, J., Bergstrom, G. C., Brueggeman, R., Horsley, R. D., and Schwarz, P. B. 2018a. Malting of Fusarium head blightinfected rye (Secale cereale): Growth of Fusarium graminearum, trichothecene production, and the impact on malt quality. Toxins (Basel) 10:369.

Jin, Z., Zhou, B., Gillespie, J., Gross, T., Barr, J., Simsek, S., Brueggeman, R., and Schwarz, P. B. 2018b. Production of deoxynivalenol (DON) and DON-3-glucoside during the malting of Fusarium infected hard red spring wheat. Food Control 85:6-10.

Jones, R. K., and Mirocha, C. J. 1999. Quality parameters in small grains from Minnesota affected by fusarium head blight. Plant Dis. 83:506511.

Knight, N. L., and Sutherland, M. W. 2011. A rapid differential staining technique for Fusarium pseudograminearum in cereal tissues during crown rot infections. Plant Pathol. 60:1140-1143.

Kostelanska, M., Hajslova, J., Zachariasova, M., Malachova, A., Kalachova, K., Poustka, J., Fiala, J., Scott, P. M., Berthiller, F., and Krska, R. 2009. Occurrence of deoxynivalenol and its major conjugate, deoxynivalenol-3-glucoside, in beer and some brewing intermediates. J. Agric. Food Chem. 57:3187-3194.

Laca, A., Mousia, Z., Díaz, M., Webb, C., and Pandiella, S. S. 2006. Distribution of microbial contamination within cereal grains. J. Food Eng. 72:332-338

Laitila, A., Sarlin, T., Raulio, M., Wilhelmson, A., Kotaviita, E., Huttunen, T., and Juvonen, R. 2011. Yeasts in malting, with special emphasis on Wickerhamomyces anomalus (synonym Pichia anomala). Antonie Leeuwenhoek 99:75-84.

Lancova, K., Hajslova, J., Poustka, J., Krplova, A., Zachariasova, M., Dostalek, P., and Sachambula, L. 2008. Transfer of Fusarium mycotoxins and 'masked' deoxynivalenol (deoxynivalenol-3-glucoside) from field barley through malt to beer. Food Addit. Contam. Part A 25:732-744.

Langevin, F., Eudes, F., and Comeau, A. 2004. Effect of trichothecenes produced by Fusarium graminearum during Fusarium head blight development in six cereal species. Eur. J. Plant Pathol. 110:735-746.

Lewandowski, S. M., Bushnell, W. R., and Evans, C. K. 2006. Distribution of mycelial colonies and lesions in field-grown barley inoculated with Fusarium graminearum. Phytopathology 96:567-581.

Moldenhauer, J., Pretorius, Z. A., Moerschbacher, B. M., Prins, R., and Van Der Westhuizen, A. J. 2008. Histopathology and PR-protein markers provide insight into adult plant resistance to stripe rust of wheat. Mol. Plant Pathol. 9:137-145.

Olkku, J., Salmenkallio-Marttila, M., Swiens, H., and Home, S. 2005. Connection between structure and quality of barley husk. J. Am. Soc. Brew. Chem. 63:17-22.

Packa, D., Jackowiak, H., Góral, T., Wiwart, M., and Perkowski, J. 2008. Scanning electron microscopy of Fusarium-infected kernels of winter triticale ( $\times$ Triticosecale Wittmack). Seed Sci. Biotechnol. 2:27-31.

Peters, J., van Dam, R., van Doorn, R., Katerere, D., Berthiller, F., Haasnoot, W., and Nielen, M. W. F. 2017. Mycotoxin profiling of 1000 beer samples with a special focus on craft beer. PLoS One 12:e0185887.

Pritsch, C., Muehlbauer, G. J., Bushnell, W. R., Somers, D. A., and Vance, C. P. 2000. Fungal development and induction of defense response genes during early infection of wheat spikes by Fusarium graminearum. Mol. Plant-Microbe Interact. 13:159-169.

Prom, L. K., Horsley, R. D., Steffenson, B. J., and Schwarz, P. B. 1999. Development of Fusarium head blight and accumulation of deoxynivalenol in barley sampled at different growth stages. J. Am. Soc. Brew. Chem. 57:60-63.

Puri, K. D., and Zhong, S. 2010. The 3ADON population of Fusarium graminearum found in North Dakota is more aggressive and produces a higher level of DON than the prevalent 15ADON population in spring wheat. Phytopathology 100:1007-1014.

Schwarz, P. B. 2003. Impact of Fusarium Height Blight on malting and brewing quality of barley. Pages 395-419 in: Fusarium Height Blight of Wheat and Barley. K. Leonard and W. Bushnell, eds. American Phytopathological Society, St. Paul, MN, U.S.A.

Schwarz, P. B. 2017. Fusarium head blight and deoxynivalenol in malting and brewing: Successes and future challenges. Trop. Plant Pathol. 42:153-164.

Schwarz, P. B., Casper, H. H., and Beattie, S. 1995. Fate and development of naturally-occurring Fusarium mycotoxins during malting and brewing. J. Am. Soc. Brew. Chem. 53:121-127.

Schwarz, P. B., Horsley, R. D., Steffenson, B. J., Salas, B., and Barr, J. 2006. Quality risks associated with the utilization of Fusarium head blight infected malting barley. J. Am. Soc. Brew. Chem. 64:1-7.

Scott, P. M. 1996. Mycotoxins transmitted into beer from contaminated grains during brewing. J. AOAC Int. 79:875-882.

Solanki, S., Ameen, G., Borowicz, P., and Brueggeman, R. S. 2019. Shedding light on penetration of cereal host stomata by wheat stem rust using improved methodology. Sci. Rep. 9:7939.

Solanki, S., Ameen, G., Zhao, J., Flaten, J., Borowicz, P., and Brueggeman, R. S. 2020. Visualization of spatial gene expression in plants by modified RNAscope fluorescent in situ hybridization. Plant Methods 16:71.

Stack, R. W. 1999. A summary of the USA situation on FHB. Pages 1617 in: Canadian Workshop on Fusarium Head Blight/Colloque Canadien Sur La Fusariose, Winnipeg, MB, Canada.

Strange, R. N., and Smith, H. 1971. A fungal growth stimulant in anthers which predisposes wheat to attack by Fusarium graminearum. Physiol. Plant Pathol. 1:141-144, IN5, 145-150.

Trail, F. 2009. For blighted waves of grain: Fusarium graminearum in the postgenomics era. Plant Physiol. 149:103-110.

Varga, E., Malachova, A., Schwartz, H., Krska, R., and Berthiller, F. 2013. Survey of deoxynivalenol and its conjugates deoxynivalenol-3glucoside and 3-acetyl-deoxynivalenol in 374 beer samples. Food Addit. Contam. Part A 30:137-146.

Vegi, A., Schwarz, P., and Wolf-Hall, C. E. 2011. Quantification of Tri5 gene, expression, and deoxynivalenol production during the malting of barley. Int. J. Food Microbiol. 150:150-156.

Vidal, A., Sanchis, V., Ramos, A. J., and Marín, S. 2016. The fate of deoxynivalenol through wheat processing to food products. Curr. Opin. Food Sci. 11:34-39.

Wan, J., Zhong, S., Schwarz, P., Chen, B., and Rao, J. 2018. Influence of oil phase composition on the antifungal and mycotoxin inhibitory activity of clove oil nanoemulsions. Food Funct. 9:2872-2882.

Wanjiru, W. M., Zhensheng, K., and Buchenauer, H. 2002. Importance of cell wall degrading enzymes produced by Fusarium graminearum during infection of wheat heads. Eur. J. Plant Pathol. 108:803-810.

Wu, Q., Kuča, K., Humpf, H. U., Klímová, B., and Cramer, B. 2017. Fate of deoxynivalenol and deoxynivalenol-3-glucoside during cereal-based thermal food processing: A review study. Mycotoxin Res. 33:79-91.

Zheng, Y., and Wang, Z. 2011. Contrast observation and investigation of wheat endosperm transfer cells and nucellar projection transfer cells. Plant Cell Rep. 30:1281-1288. 